\title{
Análise das Interações Sociais entre os Participantes de um Curso EaD: Uma Revisão Sistemática da Literatura
}

\author{
Francisco S. Sousa Neto, José Francisco M. Netto, Dhanielly P. R. de Lima \\ Programa de Pós-Graduação em Informática (PPGI) - Universidade Federal do \\ Amazonas (UFAM) - Manaus - AM - Brasil \\ \{francisconeto, jnetto, dhanielly\}@icomp.ufam.edu.br
}

\begin{abstract}
Nowadays the advance of information technology growing facility of your use, has propitiated in several advance in more areas of knowledge, for example in Distance Education. In this form, this paper presents a Systematic Literature Review (SLR) with proposal to identify and analyze the methods that existing that further the education, with finality the monitoring of students interactions in Learning Management System (LMS). Results of SLR showing the search realized in this ambit use or define methods of Distance Education that being explored and can guide to support future research.
\end{abstract}

Resumo. Atualmente, o avanço da tecnologia da informação e a crescente facilidade de sua utilização têm propiciado diversos avanços nas mais diversas áreas do conhecimento como, por exemplo, na Educação a Distância (EaD). Desta forma, este artigo apresenta uma Revisão Sistemática da Literatura (RSL) com o propósito de identificar e analisar os métodos existentes que promovem a educação, tendo como finalidade o acompanhamento das interações dos estudantes nos Ambientes Virtuais de Aprendizagem (AVA). Os resultados da RSL mostram que as pesquisas realizadas neste âmbito utilizam ou definem métodos para EaD que ainda estão sendo explorados e podem nortear e apoiar pesquisas futuras.

\section{Introdução}

Atualmente, a Educação a Distância $(\mathrm{EaD})$ vem ganhando espaço no campo educacional como um novo processo de aprender e ensinar. Esta modalidade de ensinoaprendizagem se apresenta como uma metodologia inovadora para suprir algumas lacunas na educação tradicional, devido aos diversos contextos sociais em que as pessoas vivem.

De acordo com Santos e Oliveira (2011), essa modalidade de ensino rompe a relação face a face entre professores e alunos e, o processo de ensino e aprendizagem ocorrem em ambientes que transcendem o espaço da sala de aula tradicional, realizandose em outros espaços e tempos que diferem dos marcados pelas escolas convencionais, atendendo as demandas cada vez mais crescentes de segmentos diferenciados da sociedade.

A EaD propõe diversas características específicas no processo de acompanhamento do desempenho dos alunos, dentre elas, destacamos algumas características do Modelo 3C de Colaboração, que envolve a comunicação, coordenação e cooperação. Segundo Pimentel et al. (2006), a comunicação se realiza por meio da 
troca de mensagens; a coordenação se realiza por meio do gerenciamento de pessoas, atividades e recursos; e a cooperação se realiza por meio de operações num espaço compartilhado para a execução das tarefas.

Podemos citar como exemplo do modelo 3C aplicado ao Ambiente Virtual de Aprendizagem (AVA), a ferramenta de comunicação: bate-papo, cujo objetivo é possibilitar a troca de mensagens entre os membros de um grupo, além disso, ela também contém elementos de coordenação e cooperação que são necessários para organizar e documentar a comunicação (FUKS et al. 2007).

Além do modelo $3 \mathrm{C}$ outra característica importante no processo educacional é a interação, que pode ser percebida pelo ciclo que engloba o contexto colaborativo, coordenativo e cooperativo por meio da interatividade dos alunos e professores no AVA.

Nessa perspectiva, alguns trabalhos têm um foco especial no aprendizado mais eficiente ou mais atraente através das interações aluno-professor, aluno-aluno. Para fundamentar essa pesquisa buscamos respaldo em elementos teóricos e metodológicos advindos dos trabalhos de Campana (2008); Lima et al. (2014); Soller (2001); Jaques (1997); Boff (2008); Bakharia \& Dawson (2011); Bremgartner e Netto, (2011); Mora et.al, (2014); Lima e Netto (2015) entre outros.

Contudo, a EaD apresenta-nos algumas peculiaridades no âmbito educacional, com isso, nota-se que esta modalidade de ensinar e aprender introduz uma nova concepção educacional. Nesse sentido, este novo processo de ensinar, rompe a idealização presencial no processo de ensino-aprendizagem. Consequentemente, a Educação a Distância surge como um novo ato pedagógico que não concentra reponsabilidade no professor, como era percebido no modelo de ensino presencial.

Com base nessas informações e nos trabalhos abordados anteriormente, esta Revisão Sistemática da Literatura (RSL) tem como objetivo principal investigar, identificar e analisar o estado da arte atual, no qual se encontram as pesquisas que contemplam o monitoramento e o acompanhamento das interações dos alunos nos Ambientes Virtuais de Aprendizagem. O objetivo é que os resultados adquiridos direcionem para desenvolvimento de trabalhos futuros. Nesse sentindo, os trabalhos mais importantes foram categorizados e analisados, especificando quais técnicas e métricas foram e estão sendo utilizadas nas pesquisas que referenciam o ensino e aprendizagem nos AVAs.

Para relatar esta RSL, além da seção de introdução, o artigo está estruturado da seguinte maneira: nas seções 2 e 3 é apresentada a fundamentação teórica. A Seção 4 discorre sobre o protocolo utilizado na RSL, identificando as questões de pesquisa. Nas seções 5 e 6 são apresentados os resultados advindos do processo empregado na RSL, assim fazendo menção as características dos trabalhos. Na Seção 7 é apresentada uma avaliação crítica dos trabalhos apontando as características dos mesmos. Por fim, a Seção 8 conclui o trabalho, apontando as contribuições, limitações e trabalhos futuros.

\section{A Importância das Interações no Processo do Ensino-Aprendizagem}

A análise das interações no processo do Ensino a Distância surge para ampliar a prática educacional pedagógica, e para que isso se torne uma realidade é necessário promover 
situações em que os alunos se defrontem com as diversas maneiras de aprender. Por isso, percebe-se que a interação social contribui no processo do conhecimento como construção do saber, interativo, interpessoal e participativo. Além disso, segundo Siebra et al. (2006) a análise de interação é importante para descobrir mais sobre o aluno, como por exemplo, suas dificuldades ou dúvidas.

A interação que ocorre na $\mathrm{EaD}$ deve ser vista como um fenômeno pedagógico e não simplesmente como uma mera questão de distância geográfica. A distância física conduz uma lacuna na comunicação, um espaço psicológico de concepções errôneas potenciais entre instrutores e alunos, que precisa ser suplantado por técnicas especiais de ensino. A superação da distância se dá por meio de procedimentos diferenciadores na elaboração da instrução e na facilitação da interação (MOORE, 2007).

Segundo Messa (2010), o processo de ensino-aprendizagem no âmbito da $\mathrm{EaD}$ contemporânea, em especial nos AVAs, está relacionada à utilização do material didático e das Tecnologias da Informação e Comunicação (TICs) disponíveis à mediação do tutor e à interação entre os alunos.

Além disso, os cursos nessa modalidade de ensino promovem a interação social entre o aluno e o professor. Desta forma, percebe-se que grandes benefícios surgem com o uso da Internet e das mídias digitais. Entretanto é notório alguns desafios para este método de aprendizagem, no qual é possível perceber a importância do acompanhamento do aluno para o professor. De acordo com Bremgartner e Netto (2011), há possibilidade de interação entre alunos e professores, compartilhando o conhecimento, devido os avanços das Tecnologias Digitais de Informação e Comunicação (TDICs) e da procura por plataformas educacionais.

De acordo com Silva e Navarro (2012), a dinâmica ensino-aprendizagem que envolve a interação professor-aluno deve abranger todos os aspectos, englobando assim as suas condições de vida, sua relação com a escola, a percepção e a compreensão do conhecimento sistematizado a ser estudado. Há diversas maneiras de ensinar e aprender e estão fortemente relacionadas nas ações efetuadas pelos mediadores em um AVA. Todavia, a interação entre os participantes promove a transformação no processo de ensino-aprendizagem.

Para Machado e Kazuko (2009), a EaD é complexa, visto que não demanda apenas em mensurar a quantidade de alunos que atingem ou mesmo a quantidade de interação que se tem, mas implica em uma reflexão, em seus parâmetros pedagógicos, sobre suas intencionalidades, já que poderão servir tanto ao ajustamento quanto para desenvolvimento educacional social.

Portanto, a aprendizagem não se dá pela simples transmissão de algo que está fora, mas sim, depende do desequilíbrio cognitivo, o qual é provocado num processo de interação, da ação do sujeito sobre o objeto do conhecimento, dos esquemas de significação que possibilitam o estabelecimento de relações com o novo equilíbrio (PINTO et al. 2002). 


\section{Intervenções Pedagógicas como Processo Colaborativo e Avaliativo na Educação a Distância}

As intervenções pedagógicas vem se tornando práticas educadoras que ajudam no processo de ensino-aprendizagem. Dessa forma, é possível por meio delas realizar uma analise do desenvolvimento ou o regresso do estudante. Todavia, podemos considerar que a interação é um ato comunicativo, que envolve seres humanos cujo objetivo é a comunicação interpretada como um ato interativo.

De acordo com Fuks et al. (2005), a comunicação envolve a negociação de compromissos e o conhecimento. Através da coordenação o grupo lida com conflitos e se organiza para evitar que os esforços de comunicação e de cooperação sejam perdidos. A Educação a Distância incrementada por recursos que permitem a comunicação e a realização de atividades promovem a construção de uma cultura informatizada e um saber cooperativo e colaborativo, por ações que privilegiam a interação (LIMA e SAUER, 2006).

Atualmente, inúmeros trabalhos estão sendo realizados na área da Informática Educacional, sendo notório o desenvolvimento de protótipos para acompanhar as interações nos fóruns de discussão, mensagens, chats, wikis etc. Além disso, constatase, igualmente, que há poucas pesquisas na área da Educação visando as intervenções pedagógicas na EaD. A EaD segundo Nova e Alves (2003), surge como uma modalidade de ensino-aprendizagem que possibilita a mediação, por intermédio dos suportes tecnológicos, digitais e de rede, seja inserida em sistemas de ensino presenciais, mistos ou completamente realizada através da distância física.

De acordo com Gerosa et al. (2003), alguns fatores citados anteriormente ajudam o docente a coordenar os aprendizes, podendo intervir a tempo quando a discussão começar a tomar rumos não desejados e podem avaliar a evolução da turma e do curso. Estes fatores também podem ser utilizados para guiar os aprendizes durante sua atuação nos fóruns e para a construção de filtros e mecanismos de coordenação que evitem antecipadamente os erros.

Contudo, a interação social corresponde a uma parte cada vez mais significativa da aprendizagem, pois cada vez mais os estudantes se sentem motivados a aprender na interação com seus pares. Essa característica do estudante clama, então, por práticas pedagógicas baseadas na colaboração.

A colaboração observada no ambiente virtual manifesta-se como um fenômeno que ocorre tanto entre os alunos quanto entre aluno e professor. O desejo de colaborar, entre os alunos, evidencia-se muito claramente entre os iniciantes que, repetidas vezes, manifestam seu interesse em estabelecer trocas e parcerias com seus colegas. A fala do aluno, dirigindo-se por e-mail aos seus colegas, representa esse interesse de troca de experiências na área de conhecimento do curso (Mülbert et al. 2006).

\section{Protocolo da Revisão Sistemática}

Segundo os critérios de Kitchenham e Charters (2007), que descrevem os passos necessários para a condução de uma RSL, buscamos encontrar pesquisas atuais e relevantes que contribuam para a realização de futuras pesquisas, que possam direcionar o ensino e aprendizagem no contexto da EaD. Além disso, foram analisadas publicações 
científicas, com o propósito de caracterizar e identificar técnicas utilizadas para desenvolver sistemas que auxiliem no ensino e na aprendizagem em AVAs.

A RSL é uma técnica de pesquisa baseada em evidências da literatura científica, conduzida formalmente, seguindo as fases de um protocolo bem definido. Tais fases incluem a realização de atividades de planejamento, de execução e de resultados a fim de responder às questões de pesquisa definidas na Seção 4. As buscas dos artigos foram realizadas por meio da submissão de strings às bases de dados indexadas em máquinas de busca digitais.

\subsection{Objetivo}

Esta RSL tem como objetivo principal seguir o protocolo definido pelo GQM (GoalQuestion-Metric) desenvolvido por Basili et al. (1994), no qual define alguns métodos a serem coletadas a fim de atender alguns objetivos. Os objetivos desta RSL estão destacados no Quadro 1.

Quadro 1. Objetivo conforme paradigma GQM

\begin{tabular}{|l|l|}
\hline ANALISAR & Publicações científicas. \\
\hline COM O PROPÓSITO DE & Caracterizar e analisar. \\
\hline EM RELAÇÃO A & $\begin{array}{l}\text { Realizar um levantamento do estado da arte existente nas diversas } \\
\text { literaturas, que tenham como finalidade analisar as interações } \\
\text { sociais entre os participantes de um curso EaD por intermédio de } \\
\text { uma abordagem multiagente. }\end{array}$ \\
\hline DO PONTO DE VISTA DOS & Dos pesquisadores. \\
\hline NO CONTEXTO & Acadêmico. \\
\hline
\end{tabular}

\subsection{Questões da Pesquisa}

A RSL apresentada neste artigo tem como finalidade responder as questões de pesquisa que são apresentadas nas Subseções 4.2.1 e 4.2.2, com o propósito de atrair estudos similares a temática abordada.

\subsubsection{Questão Principal da Pesquisa}

Com o propósito identificar os trabalhos que analisam as interações dos participantes de um AVA com o objetivo de auxiliar os professores a coordenarem as suas ações; essa RSL apresenta como questão principal de pesquisa:

Quais os trabalhos que analisam as interações sociais entre os estudantes em um Ambiente Virtual de Aprendizagem e quais os dados coletados e critérios utilizados por esses trabalhos nas análises?

\subsubsection{Questões Secundárias da Pesquisa}

A fim de responder a questão principal de pesquisa e de identificar quais as características, técnicas e critérios utilizados nos trabalhos, as questões secundárias da pesquisa são:

- Quais foram os requisitos considerados para analisar as interações entre os participantes? 
- Quais as técnicas e os métodos utilizados na coleta e análise das interações sociais entre os estudantes?

\subsection{Estratégias de Busca}

As buscas foram realizadas nas bibliotecas digitais: SCOPUS, IEEE Xplore e ACM Digital Library. Já as buscas manuais foram realizadas em eventos e conferências que atendem os requisitos para esta pesquisa, entre eles destacamos: Simpósio Brasileiro de Informática na Educação (SBIE), Simpósio Brasileiro de Sistemas Colaborativos (SBSC), Conferência Internacional sobre Informática na Educação (TISE) e Workshop de Informática na Escola (WIE).

\subsection{Idiomas}

Os idiomas escolhidos para realizar esta busca nas bibliotecas digitais e nas buscas manuais foram o Inglês e o Português, sendo que o Inglês foi utilizado nas bibliotecas por ser o idioma adotado na maioria das conferências e periódicos existentes atualmente que estão relacionados com o tema desta pesquisa. Para as buscas manuais, o idioma utilizado foi o idioma adotado pela própria conferência/evento, que foram citados na Seção 4.3.

\subsection{Expressão de Busca}

A string geral, submetida às bibliotecas digitais é apresentada a seguir, no formato de expressão lógica e utiliza os operadores $O R$ e $A N D$. Ela foi devidamente ajustada para cada mecanismo de busca e a sua estrutura foi elaborada de acordo com as características e especificidades da string. Desta forma a string de busca utilizada foi:

((\{virtual learning environment\} OR \{learning management system\} OR \{E-learning\} $O R$ \{moodle\} OR \{interactions relations groups\} OR \{interaction analysis groups\} OR \{social network analysis\} OR \{Timeline\}) AND (\{multi-agents systems\} OR \{intelligent agent\})).

\subsection{Critérios de Inclusão e Exclusão}

Com o propósito de eliminar estudos considerados desnecessários para as questões de pesquisa, as seguintes restrições foram adotadas: artigos que não estavam escritos na língua inglesa ou portuguesa, artigos duplicados, artigos sem a versão completa e artigos que não foram publicados em periódicos ou anais de conferências.

Com o intuito de facilitar a busca e a seleção desses artigos, foram definidos alguns critérios de inclusão e exclusão, os critérios adotados encontram-se no Quadro 2:

\section{Quadro 2: Critérios de Inclusão e Exclusão}

\begin{tabular}{|l|l|}
\hline \multicolumn{1}{|c|}{ Critérios de Inclusão (CI) } & \multicolumn{1}{|c|}{ Critérios de Exclusão (CE) } \\
\hline CI1: Artigos disponíveis na íntegra para download. & $\begin{array}{l}\text { CE1: Publicações que não atendem os critérios de } \\
\text { inclusão. } \\
\text { CI2: Artigos de conferências ou periódicos. }\end{array}$ \\
$\begin{array}{l}\text { CI3: Podem ser selecionadas publicações que } \\
\text { envolvam Sistemas Multiagente no processo de } \\
\text { ensino e aprendizagem em ambientes virtuais. }\end{array}$ & $\begin{array}{l}\text { ou aplicação, ou seja, artigos teóricos e de } \\
\text { mapeamentos sistemáticos são desconsiderados. } \\
\text { CE3: Artigos duplicados ou pagos. }\end{array}$ \\
\hline
\end{tabular}


Ao realizar as buscas nas bibliotecas mencionadas a seguir, obtivemos um resultado total de 269 artigos, conforme apresenta a Tabela 1.

Tabela 1: Resultados Retornados através da String de Busca

\begin{tabular}{|c|c|c|c|c|c|}
\hline $\begin{array}{c}\text { Bibliotecas } \\
\text { Digitais }\end{array}$ & $\begin{array}{c}\text { String de } \\
\text { busca }\end{array}$ & Pago & $\mathbf{1}^{\mathbf{0}}$ Filtro & $\mathbf{2}^{\mathbf{o}}$ Filtro & $\mathbf{3}^{\mathbf{0}}$ Filtro \\
\hline IEEE & 170 & - & 33 & 12 & 3 \\
\hline SCOPUS & 51 & 6 & 8 & 4 & 2 \\
\hline ACM & 39 & 11 & 5 & 2 & 1 \\
\hline TOTAL & 269 & 17 & 46 & 18 & 6 \\
\hline
\end{tabular}

Ainda segundo a Tabela 1, é possível constatar que na primeira análise avaliativa foram identificados 17 artigos pagos. Após a exclusão dos artigos pagos, realizou-se uma leitura do título e do abstract (resumo) dos 252 artigos restantes, sendo que desses 252 artigos, restaram somente 46 para a próxima fase de análise. Para análise do $1^{\circ}$ filtro, realizamos uma leitura da introdução e conclusão dos 46 artigos. Para o $2^{\circ}$ filtro haviam 18 trabalhos, onde foram realizados os procedimentos do $1^{\circ}$ filtro mais a leitura parcial nas seções identificadas como relevantes para pesquisa. Todavia, para o $3^{\circ}$ filtro restaram 6 artigos que foram lidos na íntegra a fim de identificar as informações relevantes para o desenvolvimento da pesquisa.

\section{Extração de Informações}

Para a extração das informações, nos baseamos em fornecer um conjunto de respostas inerentes para cada questão de pesquisa que foram definidas na Seção 4.2. Este procedimento de extração dos dados assegura a aplicação utilizada para todos os artigos selecionados e consequentemente facilita na classificação dos mesmos, obtendo desta forma um resultado satisfatório e relevante nesse processo.

As informações extraídas dos artigos pré-selecionados para o $2^{\circ}$ filtro são detalhadas no Quadro 3 onde serão apresentadas dados analisados pelos autores. 
Quadro 3: Dados Analisados dos Artigos e Referências

\begin{tabular}{|c|c|}
\hline Dados Analisados & Referências \\
\hline $\begin{array}{l}\text { E-mail, Mensagens, Interação e } \\
\text { Comunicação }\end{array}$ & $\begin{array}{l}\text { Lima et al. (2014); Jaques, (1997); Bakharia e Dawson, (2011), } \\
\text { Nolker e Zhou, (2006); Soller, (2001); Blot, (2014); Dráždilová et } \\
\text { al. (2008), Stepanyan, (2014), Gerosa et al. (2003), Fuks et al. } \\
(2005) \text {. }\end{array}$ \\
\hline Fórum de Discussão e Tarefas & $\begin{array}{l}\text { Lima et al (2014), Bremgartner e Netto, (2011); Jaques, (1997), } \\
\text { Soller, (2001); Gerosa et al. (2003), Fuks et al. (2005). }\end{array}$ \\
\hline $\begin{array}{l}\text { Colaboração, Gerenciamento e } \\
\text { Tutoria }\end{array}$ & $\begin{array}{l}\text { Soller, (2001); Boff, (2008); Dráždilová et al. (2008), (Bentivoglio, } \\
\text { 2009), Bremgartner e Netto, (2011); Stepanyan et al. (2014); Gerosa } \\
\text { et al. (2003), Fuks et al. (2005). }\end{array}$ \\
\hline $\begin{array}{l}\text { Acompanhamento e Formação de } \\
\text { Grupos }\end{array}$ & $\begin{array}{l}\text { Lima et al (2014); Daradoumis et al. (2006); Stepanyan et al. } \\
\text { (2014); Dráždilová et al. (2008). }\end{array}$ \\
\hline
\end{tabular}

\section{Trabalhos Analisados}

Nesta seção são apresentados os trabalhos resultantes da pesquisa. Primeiramente foram pré-selecionados 18 artigos no $2^{\circ}$ filtro, onde foram aplicados os critérios de inclusão e exclusão, resultando 6 artigos a serem analisados no $3^{\circ}$ filtro. Para atingir o objetivo dessa RSL, apresentamos no Quadro 4 elementos que embasam as respostas relacionadas às questões de pesquisa abordadas anteriormente, onde foram identificadas características e métodos aplicados nestes trabalhos. Nesta perspectiva analítica dos trabalhos, foi possível analisar as diversas abordagens acerca das interações sociais nos ambientes virtuais de aprendizagem.

Quadro 4: Características e Métodos de Visualização

\begin{tabular}{|c|c|c|c|c|}
\hline \multirow{2}{*}{ Autores } & \multicolumn{4}{|c|}{ Características } \\
\cline { 2 - 5 } & $\begin{array}{c}\text { Sistemas } \\
\text { Multiagente }\end{array}$ & $\begin{array}{c}\text { Interações } \\
\text { Sociais }\end{array}$ & Plataforma & Visualização \\
\hline Lima et al.(2014) & Sim & $\begin{array}{c}\text { Aluno/Aluno } \\
\text { Professor/Aluno }\end{array}$ & Moodle & $\begin{array}{c}\text { Sociogramas/ } \\
\text { Grafos/ Tabelas }\end{array}$ \\
\hline Jaques, (1997) & Sim & Aluno/Aluno & Moodle & - \\
\hline $\begin{array}{c}\text { Rocha e Romani, } \\
(2001)\end{array}$ & - & Professor/Aluno & Moodle & Gráficos/Grafos \\
\hline $\begin{array}{c}\text { Bakharia e } \\
\text { Dawson, (2011) }\end{array}$ & - & Professor/Aluno & Moodle & $\begin{array}{c}\text { Gráficos Sociais/ } \\
\text { Sociogramas }\end{array}$ \\
\hline $\begin{array}{c}\text { Drázdilová } \text { et al. } \\
(2008)\end{array}$ & - & Aluno/Aluno & Moodle & Gráficos \\
\hline \begin{tabular}{c} 
Soller, (2001) \\
\hline
\end{tabular} & Sim & Aluno/Aluno & Moodle & - \\
\hline
\end{tabular}

O trabalho de Lima et al. (2014), apresenta uma estratégia baseada numa arquitetura multiagente, tendo como objetivo analisar as interações entre os estudantes que ocorrem nos fóruns e nas mensagens de um AVA, os resultados dessas análises são 
estruturados e apresentados ao professor sob a forma de sociogramas e de tabelas, além disso, o professor recebe alertas via e-mail dos alunos com baixa ou nenhuma interação.

Jaques (1997), apresenta um experimento utilizando uma arquitetura multiagente para realizar o monitoramento das ferramentas de comunicação em um ambiente de Ensino a Distância. Este sistema analisa as discussões que se encontram em andamento nas ferramentas de lista de discussão, chat e newsgroup, disponibilizando ao professor informações estatísticas (percentual de participação dos alunos e número de mensagens trocadas), e identificando possíveis associações nas interações, tais como, tópicos e subtópicos que interessam ao aluno e aos grupos de alunos que interagem mais entre si.

O trabalho de Rocha e Romani (2001), apresenta a ferramenta InterMap (Interaction Map), que provê um modelo de interface gráfica interativa. Com o auxílio de técnicas de visualização da informação o trabalho representa graficamente os dados das ferramentas de comunicação em ambientes de Educação a Distância na Web.

Bakharia e Dawson (2011), apresentam uma ferramenta que tem como objetivo analisar a interação no fórum entre os estudantes de um curso on-line e assim proporcionar uma representação visual de fácil interpretação da organização do grupo social nos fóruns.

Drázdilová et al. (2008), analisa através de gráficos em redes sociais os dados do sistema de e-Learning. Os dados analisados contém registros sobre as atividades dos alunos em um sistema de AVA. Identificamos também neste trabalho métodos de agrupamento e, consequentemente, foram utilizados métodos de análise de rede social. As redes sociais analisadas neste trabalho são representadas por grupos de estudantes que possuem contatos semelhantes e interagem em círculos sociais.

O trabalho de Soller (2001), proporciona uma melhor compreensão da interação do grupo e orienta a melhor forma de apoiar o processo de aprendizagem colaborativa. Todavia, propõe um quadro de princípios para o estudo e análise da interação de grupo que é construído através da combinação de diferentes aspectos e questões de colaboração e aprendizagem.

Os estudos foram selecionados de acordo com as caraterísticas e dados individuais de cada um. Os resultados obtidos com as análises destes trabalhos envolviam diferentes indivíduos, como gestores, estudantes, mediadores e tutores; podendo ser destinados a um grupo, por exemplo, uma ferramenta que auxiliasse na intervenção pedagógica do mediador, com a finalidade de melhorar a aprendizagem, utilizando técnicas de visualização e assim otimizar o trabalho do professor/mediador.

Nos trabalhos que foram selecionados, identificamos que os dados analisados foram o conteúdo do estudante, as interações sociais, o feedback ao estudante, o envio de notificações (SMS, e-mails, no AVA, etc.), diante dessa verificação percebemos que ainda há muito que ser pesquisado nesta área, enfatizando as intervenções pedagógicas em tempo real utilizando técnicas e métodos de visualização.

Nesta pesquisa foram identificados um número baixo de trabalhos que descrevem a intervenção pedagógica, no entanto, muitos trabalhos não indicam alguma possibilidade de intervir pedagogicamente, uma vez que as intervenções pedagógicas mediadas pelos professores contribuem no processo de ensino-aprendizagem. 


\section{Discussões e Desafios}

O desenvolvimento de pesquisas que envolve o processo educativo na modalidade EaD, vem fomentando análises no campo de investigação, colaboração e articulação entre os pesquisadores envolvidos com esta nova tendência educacional. Todavia, esses estudos abordam discussão de como ampliar a interação social entre os estudantes, uma vez que esse tipo de comunicação nesses ambientes torna-se dinâmico em virtude do desenvolvimento desses ambientes de aprendizagem, onde a socialização virtual é monitorada.

Neste contexto, o trabalho de Bentivoglio (2009), analisa as discussões que se encontram em andamento nas ferramentas de lista de discussão, chat e newsgroup, disponibilizando ao professor informações estatísticas (percentual de participação dos alunos e número de mensagens trocadas), e identificando possíveis associações nas interações, tais como, tópicos e subtópicos que interessam ao professor e grupos de alunos que interagem mais entre si, etc. Entretanto, a capacidade de visualizar a estrutura gráfica social e a evolução da comunidade é crucial para o sucesso e serve como um indicador antecipado do sucesso de um design de atividade, bem como as informações sobre a participação dos alunos e seu desempenho educacional.

Os artigos analisados não discutem explicitamente sobre o interesse de intervir pedagogicamente nas interações sociais entre os participantes em um curso EaD. Por exemplo, as tecnologias poderiam promover interações sociais educativas, as quais são geralmente mensuradas pela quantidade de alunos participativos de um fórum interativo no ambiente virtual de aprendizagem em tempo real. Entretanto, nos ambientes virtuais de aprendizagem podemos tecnologicamente acompanhar de maneira estratégica as interações entre os estudantes.

Considerando os diferentes níveis de interação social, Machado e Kazuko (2009), apresentam um modelo de aprendizagem colaborativa projetada para ajudar um sistema de aprendizagem colaborativa inteligente a identificar áreas problemáticas e a interação de grupo-alvo. O modelo descreve possíveis indicadores de aprendizagem colaborativa eficaz, e para cada indicador, recomenda estratégias para melhorar a interação entre pares. Este modelo de aprendizagem colaborativa dirigiu o projeto e desenvolvimento de duas ferramentas que automatizam a codificação, e auxiliam a análise da conversa de aprendizagem colaborativa e de atividade.

\section{Conclusões}

Esta pesquisa apresentou uma Revisão Sistemática da Literatura, observando o estado da arte em relação às interações sociais entre os estudantes em um Ambiente Virtual de Aprendizagem. Todavia, foram obtidos por intermédio desta RSL, resultados satisfatórios para responder a seguinte pergunta: como auxiliar o professor a identificar os diversos estágios das interações sociais entre os alunos no decorrer do curso em um Ambiente Virtual de Aprendizagem, possibilitando intervenções pedagógicas?

Desta forma, foi realizada uma coleta de artigos que dissertavam sobre as interações sociais dos estudantes. Entretanto, esta análise foi direcionada em pontos chaves, a fim de obter uma colaboração maior de alunos e professores nos experimentos 
das interações sociais nos Ambientes Virtuais de Aprendizagem, com o objetivo de identificar essas interações.

Nesta perspectiva, é necessário que a pesquisa na área de Educação a Distância promova a evolução da aprendizagem conceituando características que colaboram com o desenvolvimento educacional, socializando os alunos neste processo de ensino. Todavia, as ferramentas tecnológicas são extremamente importantes para 0 acompanhamento das interações sociais que desenvolvem a socialização em ambientes virtuais. Com isso, os artigos apresentados na RSL nos ajudaram a compreender os diversos contextos em que a pesquisa na área de computação é influente nesta modalidade de ensino.

Contudo, percebe-se que existe uma carência de estudos sobre as interações sociais entre os participantes em ambientes virtuais. Sendo assim, para trabalhos futuros pretende-se desenvolver um sistema multiagente que realize o acompanhamento das interações sociais entre cursistas de um curso $\mathrm{EaD}$ e forneça essas informações por intermédio de Data Visualization ao professor, a fim de lhe auxiliar na coordenação do curso on-line.

Por fim, esta RSL contribuiu na identificação dos principais elementos que podem ser utilizados no desenvolvimento desse sistema e a sua validação será realizada por intermédio de avaliações e testes.

\section{Agradecimentos}

Agradecemos o apoio financeiro da Fundação de Amparo à Pesquisa do Estado do Amazonas (FAPEAM) através do Edital Nº03/2015.

\section{Referências}

Bakharia, Aneesha, \& Shane Dawson. (2011). SNAPP: A Bird's-Eye View of Temporal Participant Interaction. International Conference on Learning Analytics and Knowledge LAK '11, ACM, Banff, Alberta.

Basili, Victor, Gianluigi Caldiera, \& H. Dieter Rombach. (1994). Goal Question Metric. Paradigm Encyclopedia of Software Engineering, Kansas.

Bentivoglio, C. A. (2009, November). Time Analysis of Forum Evolution as Support Tool for E-Moderating. In Intelligent Systems Design and Applications, 2009. ISDA'09. Ninth International Conference on (pp. 1131-1135). IEEE.

Blot, Guillaume, Pierre Saurel, \& Francis Rousseaux. (2014). Pattern Discovery in ELearning Courses: A Timebased Approach. International Conference on Control, Decision and Information Technologies, CoDIT, Washington, DC 20057, Estados Unidos.

Boff, Elisa (2008). Colaboração em Ambientes Inteligentes de Aprendizagem Mediada por um Agente Social Probabilístico, Tese de Doutorado, UFRGS, Porto Alegre.

Bremgartner, V., \& Netto, J. F. M. (2011). Auxílio Personalizado a Estudantes em Ambientes Virtuais de Aprendizagem Utilizando Agentes e Competências. In Anais do Simpósio Brasileiro de Informática na Educação (Vol. 1, No. 1). 
Campana, V. (2008). Agentes para Apoiar o Acompanhamento das Atividades em Ambientes Virtuais de Aprendizagem. Anais Do Simpósio Brasileiro de Informática na Educação XIX (SBIE), Fortaleza.

Daradoumis, T., Martínez-Monés, A., \& Xhafa, F. (2006). A Layered Framework for Evaluating On-line Collaborative Learning Interactions. International Journal of Human-Computer Studies, Elsevier.

Dráždilová, P., Martinovic, J., Slaninová, K., \& Snášel, V. (2008). Analysis of Relations in eLearning. In 2008 IEEE/WIC/ACM International Conference on Web Intelligence and Intelligent Agent Technology, IEEE Computer Society Washington, DC, USA.

Fuks, H., Raposo, A., Gerosa, M. A., Pimentel, M., Filippo, D., \& Lucena, C. J. P. (2007). Inter- e Intra-relações entre Comunicação, Coordenação e Cooperação. Anais do IV Simpósio Brasileiro de Sistemas Colaborativos (SBSC), Rio de Janeiro-RJ, ISBN 987-85-7669-116-7.

Fuks, H., Gerosa, M. A., Filippo, D., \& Lucena, C. J. P. (2005). Informações Estatísticas e Visuais para a Mediação de Fóruns Educacionais, Revista Brasileira de Informática na Educação (RBIE).

Gerosa, M.A., Pimentel, M.G., Fuks, H. \& Lucena, C.J.P. (2003), Coordenação de Fóruns Educacionais: Encadeamento e Categorização de Mensagens, XIV Simpósio Brasileiro de Informática na Educação, Rio de Janeiro-RJ.

Jaques, P. A. (1997). Um Experimento com Agentes de Software para Monitorar a Colaboração em Aulas Virtuais. Revista Brasileira de Informática na Educação, Comissão Especial de Informatica na Educação, Rio Grande do Sul.

Kitchenham, B., \& Charters, S. (2007). Guidelines for Performing Systematic Literature Reviews in Software Engineering Version 2.3. Engineering, Durham, UK.

Lima, D., \& Netto, J. F. (2015). Análise dos Resultados de um Sistema Multiagente que Identifica e Caracteriza as Relações Sociais dos Alunos de um Ambiente Virtual de Aprendizagem. In Anais do Simpósio Brasileiro de Informática na Educação (Vol. 26, No. 1, p. 1274).

Lima, D., Gadelha, B., Netto, J. F., \& Bremgartner, V. (2014). MoodleGroups: um Aplicativo para Identificar as Relações Sociais entre Alunos em Ambientes Virtuais de Aprendizagem. Simpósio Brasileiro de Sistemas Colaborativos. October (Vol. 6, p. 9).

Lima, I. G., Sauer, L. Z., Soares, E. M. S. (2004). Discutindo Alternativas para Ambientes de Aprendizagem de Matemática para Cursos de Engenharia. In: World Congress On Engineering And Technology Education, Anais. Engineering Education in the Changing Society, Guarujá.

Machado, S., \& Kazuko, T. (2009). Mediação Pedagógica Em Ambientes Virtuais De Aprendizagem: a Perspectiva Dos Alunos. Congresso Nacional de Educação, EDUCERE.

Messa, W. C. (2010). Utilização De Ambientes Virtuais De Aprendizagem - AVAs: A Busca por Uma Aprendizagem Significativa. Revista Brasileira de Aprendizagem 
Aberta e a Distância, (Ambientes Virtuais), pag:1-49.

Mora, N., Caballé, S., Daradoumis, T., Gañán, D., \& Barolli, L. (2014). Providing Cognitive and Social Networking Assessment to Virtualized Collaborative Learning in Engineering Courses. In Intelligent Networking and Collaborative Systems (INCoS), 2014 International Conference on (pag. 463-468). IEEE.

Moore, M. G., Kearsley, G., Galman, R., \& Mello, A. (2007). Educação a distância: uma visão integrada. Cengage Learning.

Mülbert, A. L., Girondi, A., Pereira, A. T. C., \& Nakayama, M. K. (2013). Affection and Conflict in Learning Management System Interactions. RIED. Revista Iberoamericana de Educación a Distancia.

Nolker, R., \& Zhou, L. (2006). Mapping Discussion Roles: From the Classroom to the Online Discussion Board. In Association for Information Systems - 12th Americas Conference On Information Systems, AMCIS 2006 (Vol. 7, pp. 3961-3964).

Nova, C., Alves, L. (2003), Educação a Distância: Limites e Possibilidades, Educação a Distância: Uma Nova Concepção de Aprendizado e Interatividade. São Paulo: Futura, 2003. p. 43-50.

Pimentel, M., Gerosa, M. A., Filippo, D., Raposo, A., Fuks, H., \& Lucena, C. J. P. (2006). Modelo 3C de Colaboração para o Desenvolvimento de Sistemas Colaborativos. Anais do III Simpósio Brasileiro de Sistemas Colaborativos (SBSC), Natal-RN. ISBN 85-7669-097-7.

Pinto, S. C. C., Schlemmer, E., dos Santos, C. T., Pérez, C. C., \& Rheinheimer, L. R. (2002). AVA: Um Ambiente Virtual Baseado Em Comunidades. XIII Simpósio Brasileiro de Informática na educação-SBIE2002. São Leopoldo-RS.

Romani, L. A. S., \& ROCHA, H. V. D. (2001). InterMap: Visualizando a Interação em Ambientes de Educação a Distância Baseados na Web. Encuentro Internacional De Informática En La Educación Superior, 1.

Santos, M., \& Oliveira, M. (2011). Interação e Comunicação em Educação a Distância, Interação e Comunicação em Comunidades de Aprendizagem, Santa Cruz-RN.

Siebra, S. A., Salgado, A. C., \& Tedesco, P. A. (2006). A Process for User Interaction Analysis in Collaborative Environments. Anais do III Simpósio Brasileiro de Sistemas Colaborativos (SBSC), Natal-RN. ISBN 85-7669-097-7.

Silva, O. G., \& Navarro, E. C. (2012). A Relação Professor-Aluno No Processo EnsinoAprendizagem. Revista Eletrônica Interdisciplinar.

Soller. A (2001) Supporting Social Interaction in an Intelligent Collaborative Learning System. International Journal of Artificial Intelligence in Education (IJAIED), Pittsburgh pp.40-62

Stepanyan, K., Mather, R., \& Dalrymple, R. (2014). Culture, Role And Group Work: A Social Network Analysis Perspective On An Online Collaborative Course. British Journal of Educational. 